\title{
超高层钢结构工程 BIM 应用和思考
}

\section{Application and Thinking of BIM in Super High-rise Steel Structure Engineering 周刚 陶华钧}

\author{
Gang Zhou Huajun Tao
}

浙江精工钢结构集团有限公司

中国・浙江 绍兴 312000

Zhejiang Jinggong Steel Structure Group Co.,Ltd., Shaoxing, Zhejiang, 312000, China
【摘要】超高层钢结构工程 BIM 技术在应用的过程中,除了其体制问题和环境本身有冲突 之外, 还存在一些比较大的安全隐患。因此, 文章将针对超高层钢结构工程 BIM 技术的应用 现状展开思考,提出相应的解决对策，以使超高层钢结构工程 BIM 应用得到更好的发展。

【Abstract】In the process of application of BIM technology in super high-rise steel structure engineering, there are some safety hidden dangers besides its system problems and conflicts with the environment itself. Therefore, this paper will reflect on the application status of BIM technology in super high-rise steel structure engineering and put forward corresponding countermeasures, so as to make better development of BIM application in superhigh-rise steel structure engineering.

【关键词】超高层钢结构; 结构工程; 应用

【Keywords】superhigh risesteel structure; structural engineering; application [DOI]10.36012/etr.v2i1.963

\section{1 引言}

随着经济的发展和社会科技的进步, 超高层钢结构工程 的 BIM 技术应用和思考已经越来越受到人们的重视。而超高 层钢结构指的是目前高层建筑中使用的一种新型折叠结构 ${ }^{[1]}$, 它可以针对高层建筑的使用情况和使用环境进行改变, 具有 非常强大的可行性，但是这种技术在使用中依靠信息化技术 作为引导。因此, 如何将 BIM 技术作为一种领先型技术应用 到超高层钢结构工程中, 就是文章的研究目的所在。

\section{2 超高层钢结构工程 BIM 的概述}

BIM 技术指的是建筑模型技术, 是利用信息化体制和信 息化技术，通过将周围的环境利用信息化监视控制模式进行 监督之后所形成的一种建筑模型。这种模型具有非常强大的 三维效果, 可以很好地进行全方位钢结构的查看, 有利于超高 层钢结构工程的施工 ${ }^{[2]}$ 。而超高层钢结构工程在进行全面建 设的过程中, 也可以将信息化模型技术与信息化模型概念进 行整合, 实现整体工程的有效加强。同时, 超高层钢结构工程 BIM 技术, 主要是通过服务与钢结构的骨架建设和钢结构后 期的维护上对高层钢结构工程的各个缺陷进行查找, 来有效
提高钢结构的稳定性。而 BIM 技术应用于超高层钢结构工 程, 其主要的应用方法和应用方式还是以信息化技术人员和 信息化工程人员作为主体进行引导, 通过信息化基础进行整 合来实现全面建设。这就说明超高层钢结构工程的建设和利 用, 需要人来进行指导, 也要以人为核心进行使用。

\section{3 超高层钢结构工程 BIM 的应用现状}

\section{1 建筑模型技术的环境探测没有达到最高效}

建筑模型基础的环境探测没有达到最高效, 指的就是建 筑模型技术在进行环境探测的时候，并没有将信息化技术中 的优点完全发挥出来。首先, 建筑模型技术的特点就是可以根 据环境的特点进行全面模型的绘制 [3], 使超高层钢结构工程在 施工的时候可以有效地规避风险, 加强施工效率。而目前的建 筑模型技术没有将其特点充分发挥, 主要是因为目前的环境 探测技术没有达到最高效。目前, 许多建筑模型技术对于环境 探测技术的利用都是停留在初期，并没有将核心化的信息化 基础拿出来使用。这就造成目前信息化技术的使用达不到标 准, 同时, 造成建筑模型技术环境探测效率低下, 使得超高层 钢结构工程在施工的时候存在一些缺陷无法在施工过程中规 避所有风险。 
土木建筑工程 Civil and Architectural Engineering

\section{2 超高层钢结构建筑模型技术并没有将人作} 为主导

超高层钢结构建筑模型技术虽然具备非常强大的可行 性,通过信息化技术可以实现对信息化问题的有效诊断,但是 信息化基础终究是一个以固定体制作为思考的技术，而建筑 模型技术更是要依靠信息化技术作为核心权力运行的。这就 使得建筑模型技术的运行存在一定的缺陷, 难以将自我运行 技术作为核心来进行, 所以在进行超高层建筑模型的施工时, 必须将人作为主导 ${ }^{[4]}$ 。而目前许多的大型超高层钢结构建筑 施工工程在进行建筑模型的利用时, 并没有将人作为主导工 程, 这是因为他们文啬于人力资源的使用, 并且降低了建筑化 模型技术中投入的人力,任由建筑模型技术自行进行判断。而 建筑化模型技术虽然具备非常强大的探测能力, 但是在进行 危险的判断时有可能会因为体系问题而造成危险的遗漏, 这 就有可能造成后期超高层钢结构工程施工时产生许多潜在危 害, 从而威胁人们的建筑施工安全。

\section{4 超高层钢结构工程 BIM 问题的思考对策}

\section{1 加强卫星探测技术在超高层钢结构工程的}

\section{应用}

卫星探测技术, 顾名思义就是利用 GPS 导航及多种高级 卫星探测实现对周围环境的有效探查，这种技术虽然耗费的 财力物力比较大, 但是探测精度刚好是目前建筑模型工程技 术所需要的。超高层钢结构工程施工时, 因为高层结构本身就 具备一定的不稳定性, 而同时钢结构需要很强的哲学性来规 避这些风险，所以利用卫星技术不仅可以很好地对这些超高 层钢结构工程进行有效的保障, 而且可以很好地和建筑模型 技术进行结合, 形成一个相对稳定的工程体系来辅助超高层 钢结构工程施工。而加强卫星技术在超高层钢结构工程中的 施工,一定要先确保建筑模型技术和卫星通信技术的契合性。
除此之外,还要雇佣大量的新型工作人员,加强卫生信息化技 术的使用,实现卫星信息探测的有效行程。

\section{2 将工程师作为核心,引导信息化技术的进行}

信息化技术作为建筑模型技术的根本建筑模型技术,如 果想要良好的运行, 就必须确保信息化技术的核心运行方式。 但是信息化技术长久以来，在建筑模型过程中的运用都是以 自我作为引导，这就导致信息化技术很难进行有效危险的判 断,所以必须要以工程师为核心进行信息化技术的引导, 这样 才能够提高信息化技术引导的正确率。但是以工程师作为核 心引导信息化技术会消耗比较大的人力和物力,因此,中国正 在将新型的引导方向放在通过加强信息化技术的自我思考能 力,即 $\mathrm{AI}$ 技术。将 $\mathrm{AI}$ 技术应用到信息化技术中, 可以很好地 使信息化技术具备可行性，拥有自我思考的技术和自我探测 的能力, 从而使建筑模型技术的自我运作效果和核心特色效 果具有更加强大的优势。

\section{5 结语}

综上所述, 超高层钢结构的工程施工离不开 BIM 技术的 应用, 而 BIM 技术在目前工程施工中还存在一些不足。论文 通过分析其不足提出相对应的解决对策, 以期促进 BIM 技术 的发展, 促进社会的进步。

\section{参考文献}

[1]陈夏标. 岩土工程勘察与地基设计方式研究 [ ] . 世界有色金属, 2017(23):265+267.

[2]阎瑞. 探究岩土工程勘察的常见问题及解决策略[].建筑知识, 2017(15):1.

[3] 孙殿英.岩土工程勘察质量有关问题及对策 []].世界有色金属, 2017(5):163+166.

[4]付敏.岩土工程勘察在复杂地质条件下的技术应用探讨 [J].西 部探矿工程,2019(5):26-27. 